\title{
Real time processing with low cost uncooled plane array IR camera-Application to flash non- destructive evaluation
}

\author{
By David MOURAND, Jean-Christophe BATSALE
}

L.E.P.T.-ENSAM, UMR 8508 CNRS, Esplanade des Arts and Metiers - 33405 Talence Cedex FRANCE - Phone : (33) 556845429 -Fax : (33) 556845436

E-mail : mourand@ lept-ensam.u-bordeaux.fr

\section{Abstract:}

A one-dimensional transient flash method based on infrared thermography device, allows to go back to thermal diffusivity map. However, each pixel of the temperature image presents a very noisy temporal signal. Moreover, in the case of an uncooled plane array camera, each plane array detector presents independent calibration coefficients and independent offset levels.

We present, in this framework, an identification method based on the estimation of thermal diffusivity variations around a nominal value by an asymptotic gradient expansion.

Such method and real time processing and computing devices allow to obtain the estimation result at the same time as the evolution of the experiment.

\section{Introduction}

A transient flash experiment based on infrared thermography allows to obtain a diffusivity variation map, using the asymptotic gradient method presented by Mourand and al, 1998 [1].

The aim of this work is to realise a cheap and real time Non Destructive Evaluation device (drying process control, line control, etc....) on materials of parallelipedic form and thickness about one centimeter.

To do this, we use an asymptotic gradient method with a low-cost infrared camera and this to the detriment of the measurement noise which can be processed by using a great number of measurement (see Mourand, 1998 [2]).

We have chosen an uncooled thermal imaging camera with a $240 \times 320$ ferromagnetic plane array detectors: the PalmIR 250 of RAYTHEON.

First, we will call back the principle of the identification method based on the estimation of thermal diffusivity variations around a nominal value by an asymptotic gradient expansion.

Then, we will show, that the asymptotic gradient method can be used with an uncooled thermal imaging camera as the PalmIR 250.

Then, an experiment with the uncooled thermal imaging camera is presented on a Poly Vinyl Chloride sample of different thickness generated by machining. A real time recording and computing device allows to obtain a real time estimation of the thermal diffusivity cartography.

\section{Estimation method of a variation of the thermal diffusivity around a nominal value}

In the ideal case, that is to say with an insulated sample, at initial time $(t=0)$, the temperature is assumed to be uniform $(T(x, t=0)=0)$, and it is submitted to a thermal 
excitation. If the heat transfer is supposed 1-D, then, the temperature response on the rear face $(x=L)$, to an instantaneous and uniform thermal pulse on the front face $(x=0)$, is given by (See Parker and al, 1961 [3]):

$$
T(t)=T_{\text {lim }} \cdot\left(1+2 \cdot \sum_{n=1}^{\infty}(-1)^{n} \exp \left(-\frac{n^{2} \cdot \pi \cdot 2 \cdot a \cdot t}{L^{2}}\right)\right)=T_{\text {lim }} f(a, t)
$$

From expression (1), the estimation problem of the parameter $a$ (diffusivity) is the same as the estimation of the parameter $a / L^{2}$ (characteristic frequency) when $L$ is known. Both parameters ( $a$ or $a / L^{2}$ ) will then be considered indifferently.

In the case where the average diffusivity $a_{0}$ of the material is known, and where the aim is only to estimate its local variation $\Delta a=a-a_{0}$, the temperature at the time $t_{i}: T\left(t_{i}\right)$ can be written with a first order development:

$$
T\left(t_{i}\right)=T_{\text {lim }} f\left(a_{0}, t_{i}\right)+\left.\Delta a T_{\text {lim }} \frac{\partial f}{\partial a}\right|_{\left(a_{0}, t_{i}\right)}
$$

That is to say with matrix notation, considering the vectors:

and parameters:

$$
\begin{aligned}
& \boldsymbol{T}=\left[\begin{array}{lll}
T\left(t_{1}\right) & \cdots & T\left(t_{N}\right)
\end{array}\right]^{t}, \boldsymbol{f}=\left[\begin{array}{lll}
f\left(a_{0}, t_{1}\right) & \cdots & f\left(a_{0}, t_{N}\right)
\end{array}\right]^{t}, \\
& \text { and } \frac{\partial \boldsymbol{f}}{\partial a}=\left[\begin{array}{lll}
\left.\frac{\partial f}{\partial a}\right|_{\left(a_{0}, t_{1}\right)} & \cdots & \left.\frac{\partial f}{\partial a}\right|_{\left(a_{0}, t_{N}\right)}
\end{array}\right]^{t}
\end{aligned}
$$

$$
\beta_{1}=T_{\text {lim }} \text { and } \beta_{2}=\Delta a T_{\text {lim }},
$$

The relation $(2-\mathrm{a})$ yields:

$$
\boldsymbol{T}=\left[\begin{array}{ll}
\boldsymbol{f} & \frac{\partial \boldsymbol{f}}{\partial a}
\end{array}\right]\left[\begin{array}{l}
\beta_{1} \\
\beta_{2}
\end{array}\right]=\boldsymbol{X}\left[\begin{array}{l}
\beta_{1} \\
\beta_{2}
\end{array}\right]
$$

This result leads to an excellent approximation (better than one percent) in a neighborhood of $10 \%$ around $a_{0}$ (see figure 1 ).

If the measurement noise on each component of the experimental temperature vector $\hat{\boldsymbol{T}}$ is assumed with a constant standard deviation and not correlated, in the domain of validity of the development (2-a), then, the optimal estimator of the parameters vector $\left[\begin{array}{ll}\hat{\beta}_{1} & \hat{\beta}_{2}\end{array}\right]^{t}$ is obtained by (see Beck and Arnold, 1971 [4]):

$$
\left[\begin{array}{l}
\hat{\beta}_{1} \\
\hat{\beta}_{2}
\end{array}\right]=\left(\boldsymbol{X}^{t} \boldsymbol{X}\right)^{-1} \boldsymbol{X}^{t} \hat{\boldsymbol{T}}
$$

As $\Delta a=\beta_{2} / \beta_{1}$, the variation of diffusivity can be written:

$$
\Delta \hat{a}=\frac{\left(\boldsymbol{f}^{t} \boldsymbol{f}\right)\left(\frac{\partial \boldsymbol{f}^{t}}{\partial a} \hat{\boldsymbol{T}}\right)-\left(\frac{\partial \boldsymbol{f}^{t}}{\partial a} \boldsymbol{f}\right)\left(\boldsymbol{f}^{t} \hat{\boldsymbol{T}}\right)}{\left(\frac{\partial \boldsymbol{f}^{t}}{\partial a} \frac{\partial \boldsymbol{f}}{\partial a}\right)\left(\boldsymbol{f}^{t} \hat{\boldsymbol{T}}\right)-\left(\boldsymbol{f}^{t} \frac{\partial \boldsymbol{f}}{\partial a}\right)\left(\frac{\partial \boldsymbol{f}^{t}}{\partial a} \hat{\boldsymbol{T}}\right)}
$$

The linearisation given by the expression (2) allows not only the estimation of the parameter $\Delta a$, but also allows the confidence interval of this estimation to be studied. 
An intermediate stage is the covariance matrix of the estimation on the vector $\left[\begin{array}{ll}\hat{\beta}_{1} & \hat{\beta}_{2}\end{array}\right]^{t}$, given depending on the standard deviation of the temperature measurement noise $\sigma_{T}$ :

$$
\operatorname{cov}\left[\begin{array}{l}
\hat{\beta}_{1} \\
\hat{\beta}_{2}
\end{array}\right]=\left(\boldsymbol{X}^{t} \boldsymbol{X}\right)^{-1} \sigma_{T}{ }^{2}
$$

The random variable relative to the estimation error on the parameter $\Delta a$ can be expressed as $e_{\Delta a}$. This variable is linked with random variables relative to the estimation errors on the parameters $\beta_{1}$ and $\beta_{2}$ expressed by $e_{\beta_{1}}$ and $e_{\beta_{2}}$. Since $\Delta a=\beta_{2} / \beta_{1}$ an asymptotic expansion gives:

$$
e_{\Delta a}=\left[\begin{array}{ll}
-\frac{\beta_{2}}{\beta_{1}^{2}} & \frac{1}{\beta_{1}}
\end{array}\right]\left[\begin{array}{l}
e_{\beta_{1}} \\
e_{\beta_{2}}
\end{array}\right]=\boldsymbol{Y}\left[\begin{array}{l}
e_{\beta_{1}} \\
e_{\beta_{2}}
\end{array}\right]
$$

The variance characterizing the confidence interval of the estimation of $\Delta a$ is then given in Beck and Arnold, 1971 [4]:

$$
\operatorname{var}\left(e_{\Delta a}\right)=\boldsymbol{Y} \operatorname{cov}\left[\begin{array}{l}
\hat{\beta}_{1} \\
\hat{\beta}_{2}
\end{array}\right] \boldsymbol{Y}^{t}=\sigma_{T}{ }^{2} \boldsymbol{Y}\left(\boldsymbol{X}^{t} \boldsymbol{X}\right)^{-1} \boldsymbol{Y}^{t}
$$

The expressions (3) and (5) show that the method can be used whatever the length of the vector $\hat{\boldsymbol{T}}$. Thus, the scalar products $\left(\frac{\partial^{t}}{\partial a} \hat{\boldsymbol{T}}\right)$ and $\left(\boldsymbol{f}^{t} \hat{\boldsymbol{T}}\right)$ can be incremented as and when the camera records images. This sequential method considerably eases the problems of storage and images manipulation. But this allows above all a real time signal processing, which is necessary for a Non Destructif Evaluation device.

Moreover, the asymptotic gradient method is optimized (in the least squares sense) in the validity domain of the linear approximation. Therefore, it constitutes a simple treatment of discrimination even out of the validity domain of the model described by (2a).

At last, it is not limited to the rear face transient method, without heat losses. We can model with the vectors $f$ and $\frac{\partial f}{\partial a}$ more complex responses (measured on the front face, with heat losses, etc... see Mourand, 1998) provided that the sole variability is the thermal diffusivity $\Delta a$.

\section{Application of the asymptotic gradient method to a thermal imaging camera}

We have chosen a thermal imaging camera: the PalmIR 250 of RAYTHEON. It uses a 240x320 ferromagnetic uncooled plane array detectors.

The camera which only have one sight and one video ouput, has been connected to an acquisition board in order to record and process a blast of 8 bites coded images in real time. Thus, the data coming from the camera are 240×320 matrix with 256 temperature level.

We should specify that the thermal imaging measurement noise is very important, practically as the same order as the signal amplitude. But as we have ever shown it (see [1] and [2]), the asymptotic gradient method is suitable to process very noisy thermograms.

One can conclude that for these required conditions, the gradient method is the most convenient and accurate. 
As we can see in the expression (4), this method doesn't need the absolute temperature to determine the thermal diffusivity variations. We just need a measurement proportional to the temperature. Thus, it is not necessary to calibrate the different camera's detector, or to know the material's emissivity.

The main problem of the PalmIR 250 camera is that it modifies continously the image's mean level, in order to optimize the image dynamic. Thus, if they had not measurement noise, the rear face transient pulse response would be obtained such as these presented on the figure 1.

Therefore, we have numerically studied the influence of the mean level variations of the thermal imaging camera.

In fact, only the look of the temperature response to the excitation changes during the experiment, thus, it only takes to replace the steady state temperature sensivity in the process, by that we will call the imaging temperature sensitivity (see figure 2).

Therefore, we have performed a numerical simulation of the parameter estimation of the "thermal diffusivity variation" $\Delta a$ with the imaging camera temperature response depending on the standard deviation of the measurement temperature.

\section{Experimental results}

To illustrate the theoretical results, an experiment was performed on a parallelpipedic form Poly Vinyl Chloride sample. Instead of varying the diffusivity, we varied the thickness. Indeed, in all the previous presentation the variations of the parameter $a / L^{2}$ may be analyzed instead of $a$. The tested thicknesses are respectively : $4.95 \mathrm{~mm}, 4.9$ $\mathrm{mm}, 4.8 \mathrm{~mm}, 4.6 \mathrm{~mm}$.

The experimental results were obtained with the infrared camera type PalmIR 250. The maximal scanning speed is 30 images/s, each image is composed of $320 \times 240$ pixels. Each experiment is composed of more than 1000 images recorded at a regular time interval. During the experiment, the data are recorded and processed in real time with a Matrox recording device (Sony) on a PC pentium II 233. Only the scalar products $\left(\frac{\partial f}{\partial a}^{t} \hat{\boldsymbol{T}}\right)$ and $\left(\boldsymbol{f}^{t} \hat{\boldsymbol{T}}\right)$ are incremented and no image is definitely stored.

Figure 3 presents an experimental thermogram corresponding to a pixel aimed by the camera, after a flash.

Figure 3 presents the image obtained thanks to the expression (4). One observes different zones corresponding to the different thickness of the plate. The maximum diffusivities of the image are the darkest.

Figure 4 presents the average profile taken from the last image. The good agreement between the estimated thickness and the real thickness, may be noted. Such average thickness profiles are compared with results obtained in the same conditions with a mechanical scanning camera of type AVIO TVS 2000 and previously published (see [1]). Some discrepancies (2D effects) can be attibuted to the specificities of electronic system. Somes studies to take into account such 2D effects are in study.

\section{Conclusion}

Our estimation method associated on an uncooled thermal imaging like the PalmIR 250 of RAYTHEON, constitutes a low-cost Non Destructive Evaluation device by infrared thermography. This work shows that the poor performances or the particularities of the infrared camera can be compensed by adapted data processing. 


\section{References}

[1] Mourand D., Gounot J., Batsale J.-C., New sequential method to process noisy temperature response from flash experiment measured by infrared camera, Review of scientific instruments, Vol. 69, №3, pp. 1437-1440, (1998).

[2] Mourand D., Contribution à the mise au point de méthode de Contrôle Non Destructif thermique - Traitement de signaux fortement bruités. Thèse de I'ENSAM Centre Bordeaux, (1998).

[3] Parker W. J., Jenkins W., Abott J., Flash method of determining thermal diffusivity, Heat capacity and thermal conductivity, Journal Appl. Phys., vol 32, No 9, pp 1679-1684, (1961).

[4] Beck J.V. and Arnold K. J., Parameter estimation in engineering and science,John Wiley and Sons, NY, (1977).

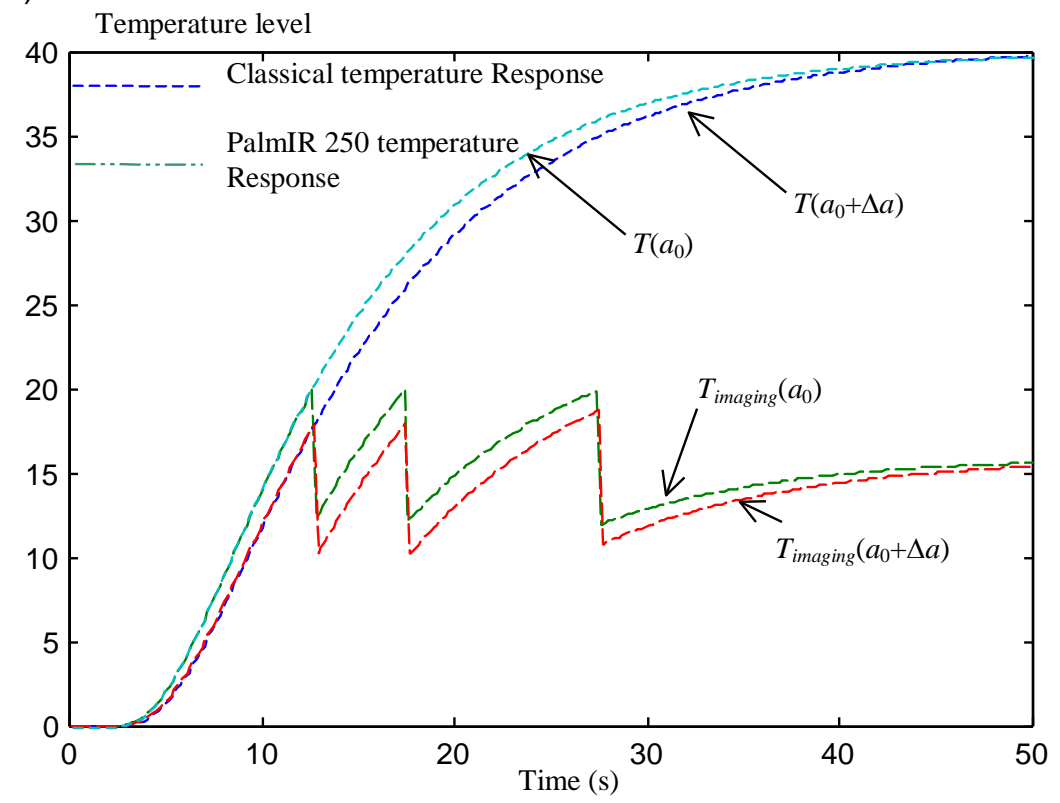

Figure 1 : Comparison of temperature response to a rear face transient method in the classical case and when the temperature is measured with the thermal imaging PalmIR 250.

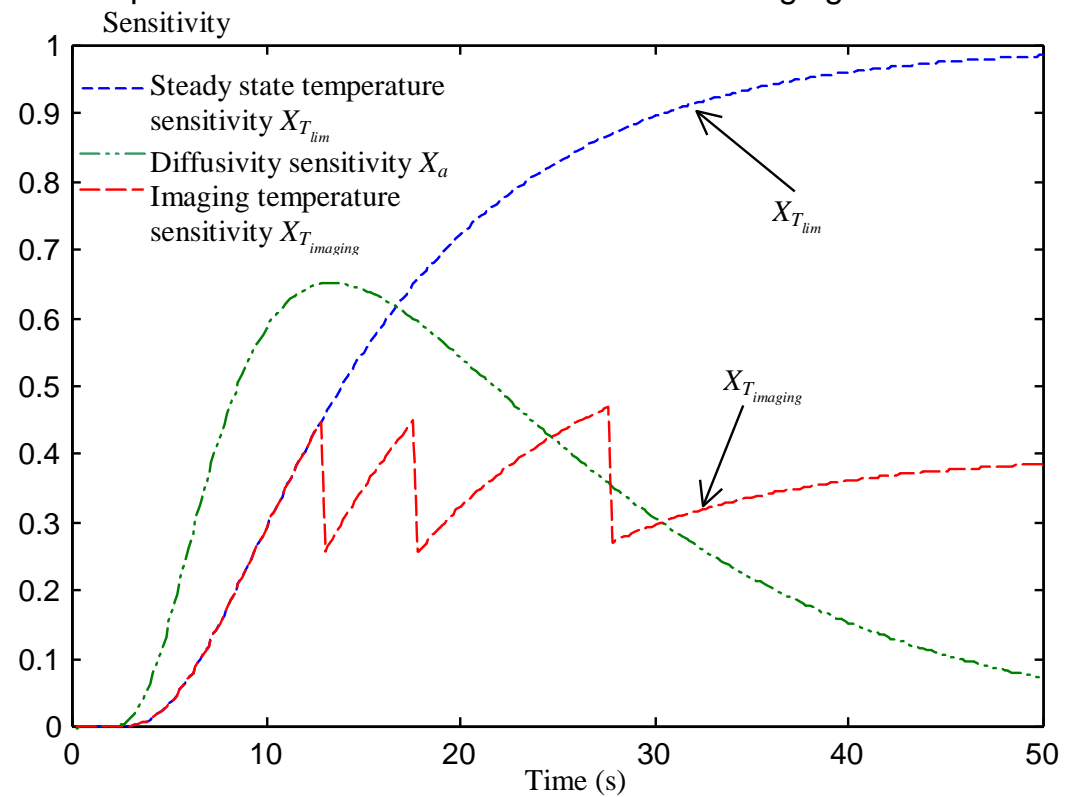

Figure 2 : Sensitivity curves numerically calculated for the steady state temperature, the thermal diffusivity, and the imaging temperature. 


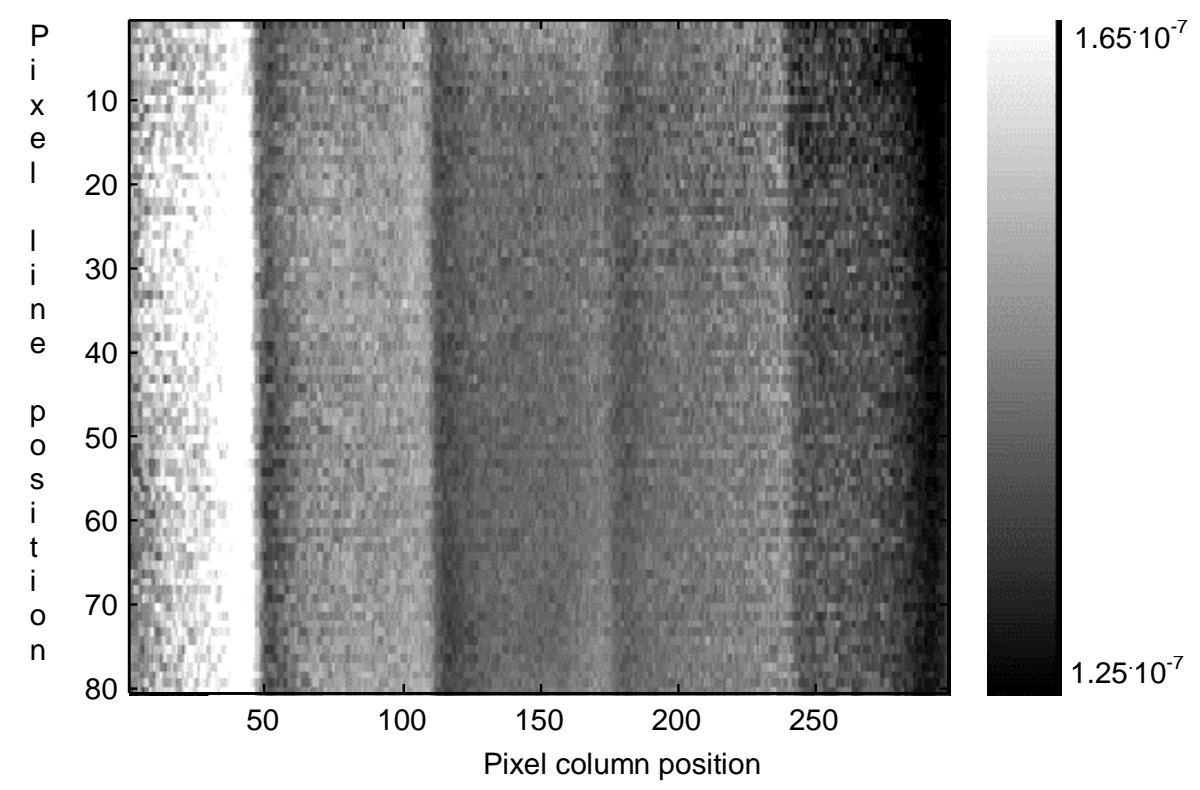

Figure 3: Thermal diffusivity map corresponding to a sample machined with different thicknesses and obtained with the PalmIR 250 and real time processing.

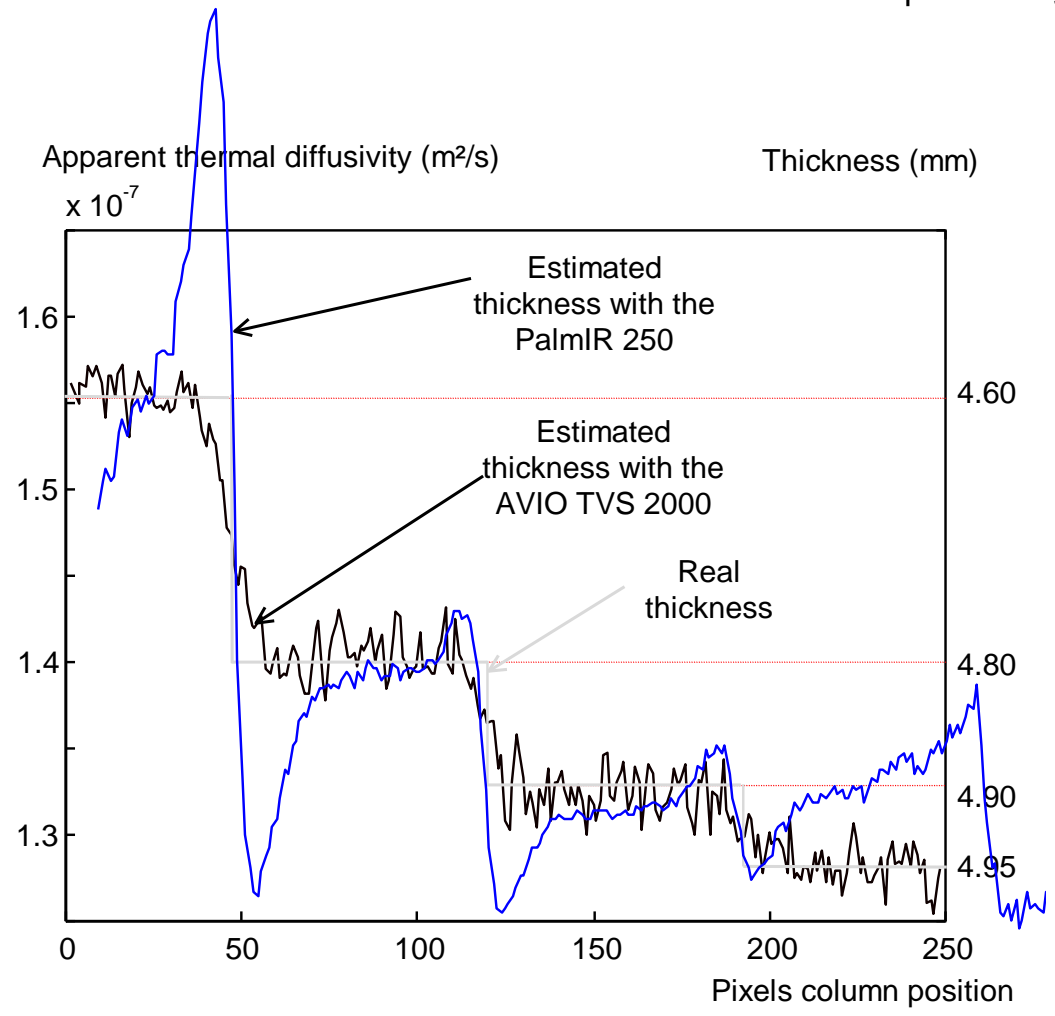

Figure 4 : Average thickness profiles obtained with the palmIR 250 camera taken from figure 3 and comparison of results obtained in the same conditions with a camera of type AVIO TVS 2000. 